\title{
Device-associated thrombus after percutaneous left atrial appendage closure: a case report and literature review
}

\section{Corresponding author:}

Iwona Świątkiewicz MD, PhD Department of Cardiology University Hospital No. 1. 9 Sklodowskiej-Curie St. 85-094 Bydgoszcz, Poland Phone +48525854023 Fax + $4852585-4024$ E-mail: iwona.swiatkiewicz@gmail.com

Medical Research Journal 2016; Volume 1, Number 1, 48-52 10.5603/MRJ.2016.0008 Copyright (C) 2016 Via Medica ISSN 2451-2591

\begin{abstract}
Introduction. In the setting of atrial fibrillation (AF), left atrial appendage (LAA) closure using the Watchman $^{\mathrm{TM}}$ device (WD) was proven to effectively prevent stroke, systemic embolism and cardiovascular death when compared with warfarin therapy. However, this procedure is potentially associated with the risk of complications, including device-attached thrombus formation.

Case presentation. We report a case of a 65 -year-old woman with permanent non-valvular AF, a history of ischemic stroke on warfarin treatment, hypertension, diabetes mellitus, heart failure with preserved left ventricular ejection fraction, labile values of the international normalized ratio, enlarged left atrium (LA) and spontaneous echo contrast in echocardiography, who was qualified for percutaneous LAA closure using the WD. The pre-procedural patient assessment indicated high thromboembolic $\left(\mathrm{CHA}_{2} \mathrm{DS}_{2}\right.$-Vasc Score $\left.=5\right)$ and bleeding risk (HAS-BLED Score $=4$ ), and optimal morphology of LAA in transesophageal echocardiography (TEE). After a successful procedure using a $30 \mathrm{~mm}$-sized WD, and despite appropriate antiplatelet and anticoagulation therapy, 6-month follow-up TEE revealed a mobile thrombus $(2.4 \mathrm{~cm} \times 0.6 \mathrm{~cm})$ at the atrial side of the WD. Therapy with intravenous heparin was started. However, a control TEE examination after one week revealed progression of the thrombus size $(4.2 \mathrm{~cm} \times 0.7 \mathrm{~cm})$ and its protrusion across the mitral valve orifice into the left ventricle. Although the patient remained asymptomatic, cardiosurgical excision of the thrombus was performed due to high risk of thrombus embolization.

Conclusions. Late device-associated thrombus formation after implantation of the WD remains a rare but severe complication. Formal recommendations regarding prevention and management of device-related thrombosis are still lacking. In this complex clinical setting, we suggest: 1) careful long-term echocardiographic monitoring after percutaneous LAA closure, especially in patients with permanent AF, high thromboembolic risk, large LA and dense spontaneous echo contrast in echocardiography, and 2) an individualized treatment approach.
\end{abstract}

Key words: atrial fibrillation, thromboembolic risk, left atrial appendage closure, Watchman ${ }^{\mathrm{TM}}$ device

Med Res J 2016; 1 (1): 48-52

\section{Introduction}

Left atrial appendage (LAA) occlusion using percutaneous interventional techniques has been demonstrated to be equivalent to oral anticoagulation (OA) in reducing thromboembolic events in patients with non-valvular atrial fibrillation (AF) [1-4]. The actually accepted indications for catheter-based LAA occlusion have been recently published in the EHRA/EAPCl expert consensus statement [5]. According to this document, catheter-based LAA occlusion may be considered [5]:
- as replacement for OA when OA is not possible (e.g. patients with a contraindication to $O A$, patients with increased bleeding risk under $O A$, patients who refuse $O A$ );

- as a complement to $O A$;

- as adjunct to ablation of AF, or as an alternative to $\mathrm{OA}$ even if $\mathrm{OA}$ is possible.

The Watchman ${ }^{\text {TM }}$ device (WD) (Boston Scientific Corp., Marlborough, MA, USA), a first-of-its-kind, proven alternative to long-term $O A$, has shown its effectiveness and relative safety in AF patients who do not wish or 
cannot receive OA $[1-3,6]$. Finally, the use of the WD was approved by the Food and Drug Administration on 13 March 2015. However, implantation of the WD may be potentially associated with complications related to the transseptal puncture or placement of the device, such as pericardial effusion, device embolization, post-implantation sepsis and the presence of device-related thrombus $[1-3,7,8]$.

\section{Case report}

A 65-year-old woman, with permanent non-valvu$\operatorname{lar} \mathrm{AF}$, previous ischemic stroke on warfarin therapy, medicated hypertension, diabetes mellitus, chronic heart failure with preserved left ventricular (LV) ejection fraction and labile values of the international normalized ratio (INR), was admitted to our department for the assessment of indications and feasibility of the percutaneous LAA closure. Both thromboembolic and bleeding risk of the patient were high as indicated by the $\mathrm{CHA}_{2} \mathrm{DS}_{2}$-Vasc score (5 points) and the HAS-BLED score (4 points), respectively. In transthoracic echocardiography we observed left atrial (LA) enlargement, LV hypertrophy, lack of wall motion abnormalities and preserved LV systolic function. Transesophageal echocardiography (TEE) was subsequently performed to assess the width of LAA orifice, the depth of LAA and the presence of additional lobes as well as to exclude thrombi inside the LA (Fig. 1A-1C). We observed weak spontaneous echo contrast without any thrombi in the LA. The patient was qualified for the percutaneous LAA closure based on the high thromboembolic and bleeding risk and optimal LAA morphology for percutaneous occlusion. Successful percutaneous closure of LAA using a $30 \mathrm{~mm}$-sized WD was performed by venous access and transseptal puncture, with no complications during the implantation. The correct positioning of the WD at the LAA ostium was demonstrated by both echocardiography and angiography. There was a small amount of pericardial effusion and absence of residual flow around the device using colour Doppler at the end of the procedure in TEE. The patient was on a daily dose of aspirin $100 \mathrm{mg}$ and $100 \mathrm{U} / \mathrm{kg}$ intravenous heparin was added at the beginning of the procedure, followed by low-molecular-weight heparin (enoxaparin $2 \times 80 \mathrm{mg}$ s.c.) after the implantation. The OA using warfarin was started immediately after the procedure and was planned to be continued for 45 days, maintaining the international normalized ratio (INR) values within the therapeutic range (i.e. between 2.0 and 3.0). However, the patient was discharged on low-molecular-weight heparin (enoxaparin $2 \times 80 \mathrm{mg}$ s.c.), because the target INR values were difficult to be kept (it was about 4.0). A scheduled TEE
A

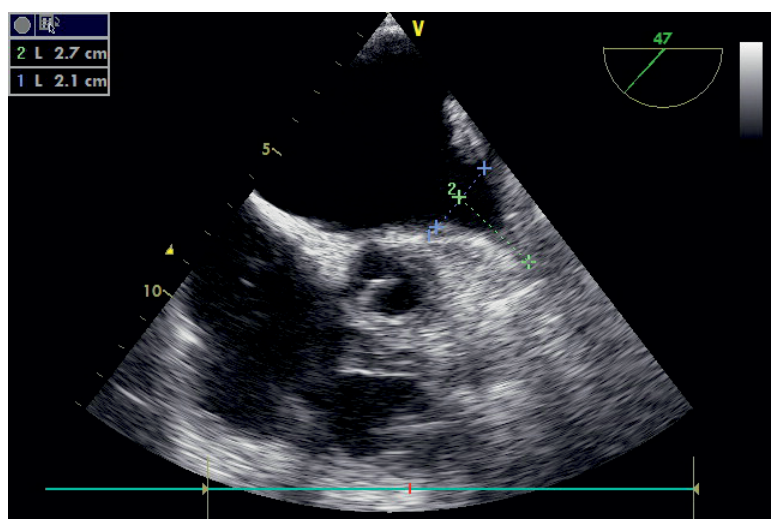

B

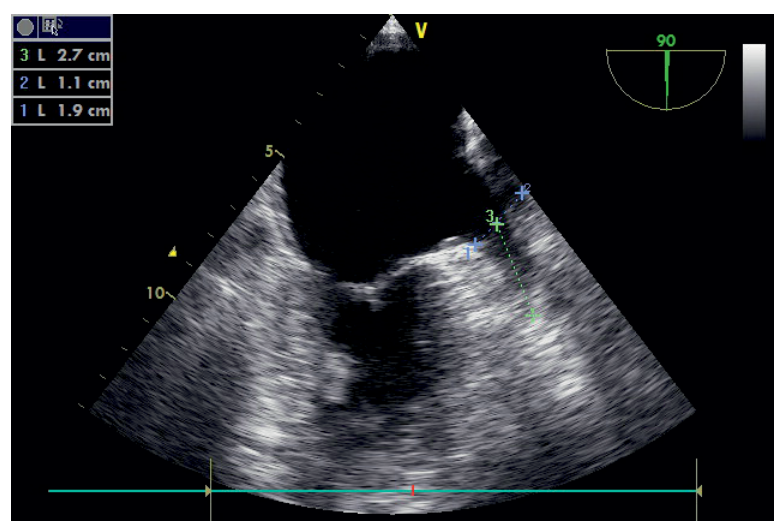

C

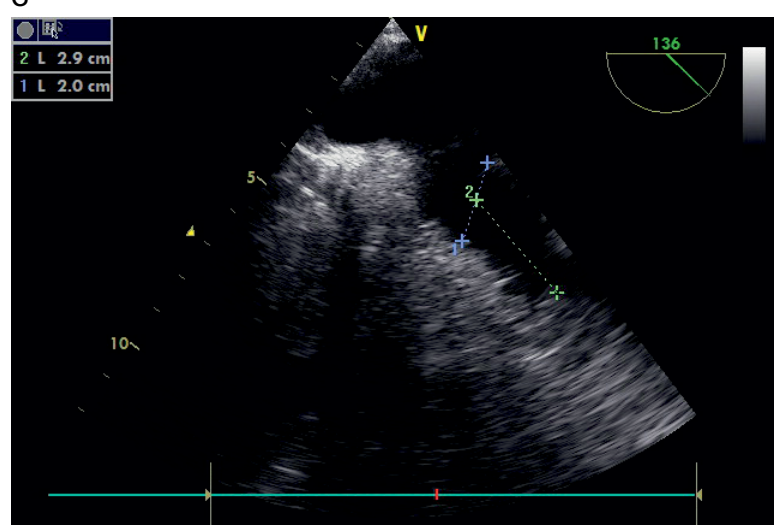

Figure 1. Left atrial appendage assessment before implantation of the Watchman ${ }^{\mathrm{TM}}$ device. Transesophageal echocardiography: mid-esophageal views (1A -47 degrees; 1B - 90 degrees; $1 \mathrm{C}-136$ degrees)

examination, performed 45 days after the procedure, showed normal position of the WD in the LAA, without residual peri-device flow, but with the presence of dense spontaneous echo contrast and small immobile echo on the atrial surface of the WD, suspected to be a small thrombus (Fig. 2A-2B). According to the results of TEE, warfarin instead of dual antiplatelet therapy (DAPT) was started, with the recommendation to maintain the INR values in the therapeutic range 


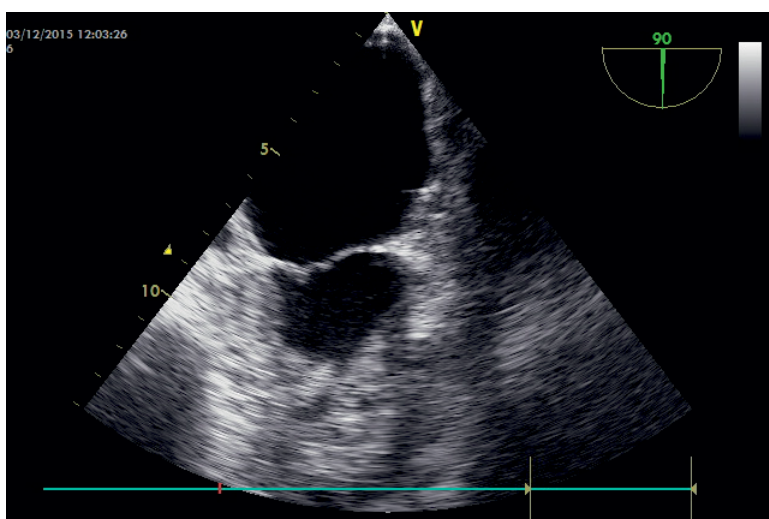

B

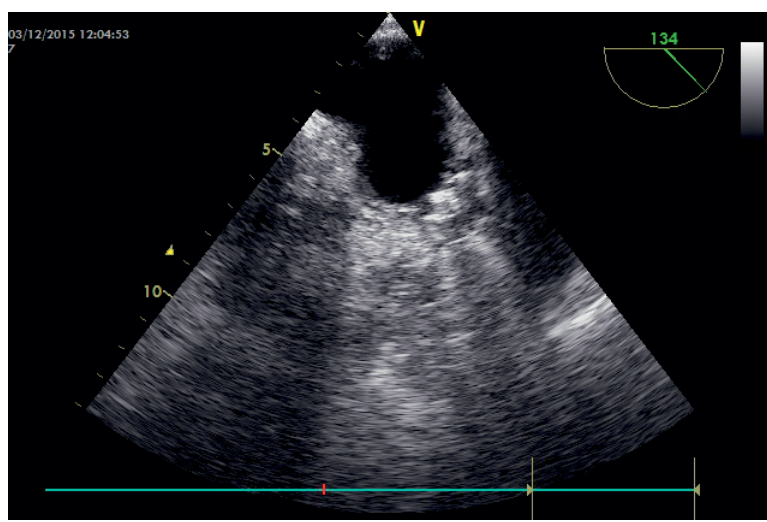

Figure 2. Watchman ${ }^{\mathrm{TM}}$ device at 45 days after implantation into the left atrial appendage. Transesophageal echocardiography: mid-esophageal views (2A - 90 degrees; $2 \mathrm{~B}-134$ degrees)

(2.0-3.0). The 6-month follow-up TEE revealed spontaneous echo contrast in the LA and a mobile thrombus $(2.4 \mathrm{~cm} \times 0.6 \mathrm{~cm})$ located at the atrial side of the WD (Fig. 3A-3B) despite the appropriate OA therapy. Because of high risk of embolization, therapy with intravenous heparin was initiated immediately (aimed to maintain the activated partial thromboplastin time values $>2.5$ times the patient's baseline control). A control TEE examination, performed one week after heparin was started, showed decreased dimensions of the thrombus $(1.7 \mathrm{~cm} \times 0.5 \mathrm{~cm})$, so heparin was replaced by low-molecular-weight heparin (enoxaparin $2 \times 80 \mathrm{mg}$ s.c.). Another TEE examination, repeated after yet another week, revealed no resolution of the thrombus, which was still mobile, floating, with greater dimensions $(4.2 \mathrm{~cm} \times 0.7 \mathrm{~cm})$, apparently originating from the screw's lodging and protruding across the mitral valve orifice into the LV (Fig. 4A-4B). Despite the fact that the patient remained completely asymptomatic, without any neurological event, cardiosurgical excision of the thrombus was performed due to high risk of thrombus embolization.
A

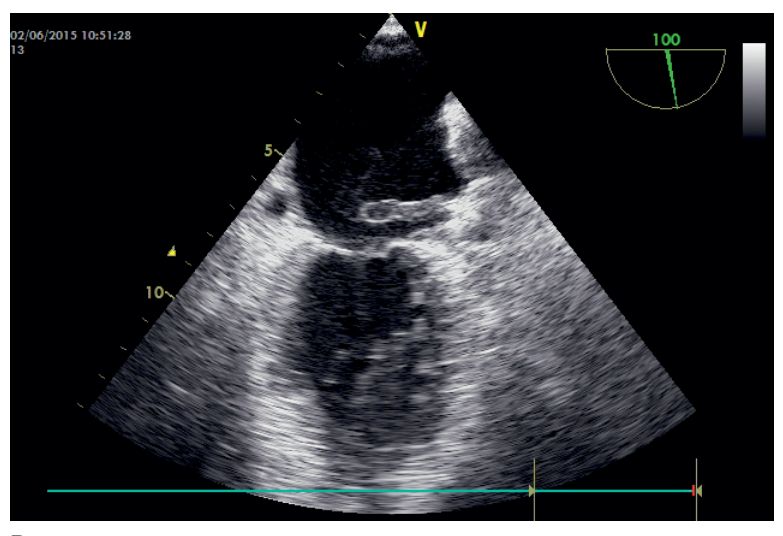

B

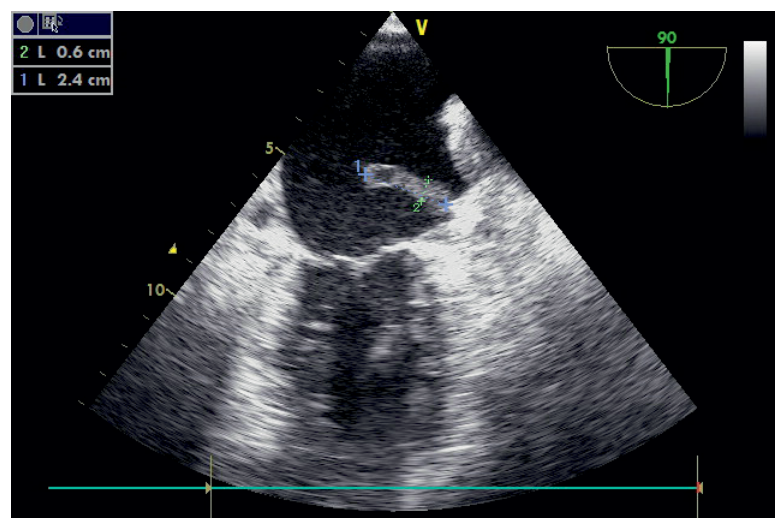

Figure 3. Thrombus formation $(2.4 \mathrm{~cm} \times 0.6 \mathrm{~cm})$ on the atrial side of the Watchman ${ }^{\mathrm{TM}}$ device 6 months after implantation. Transesophageal echocardiography: midesophageal views (3A - 100 degrees; $3 B-90$ degrees)

\section{Discussion and literature review}

LAA closure using the WD was reported to be effective for patients with AF to prevent stroke, systemic embolism and cardiovascular death, compared with warfarin therapy $[1-3,6]$. However, this procedure is associated with the risk of the formation of thrombus attached to the WD [2, 5, 7, 8]. Acute thrombosis may occur as a result of incorrect device selection or implantation (e.g. inadequate size, incorrect placement or instability of the device) $[5,7]$. Chronic thrombosis is usually observed in the follow-up TEE examination between 45 days and 3 years after the WD implantation [2, 5, 7-13]. It may be potentially triggered by incomplete closure of the LAA or increased thromboembolic risk in patients, predominantly resulting from the older age and the presence of multiple comorbidities $[2,5,7,8]$. Antiplatelet and antithrombotic treatment introduced in patients undergoing catheter-based LAA occlusion is aimed to prevent device-related thrombus formation. In line with the protocol of the PROTECT AF-trial, the usually used in clinical practice peri- and post-procedural 
A

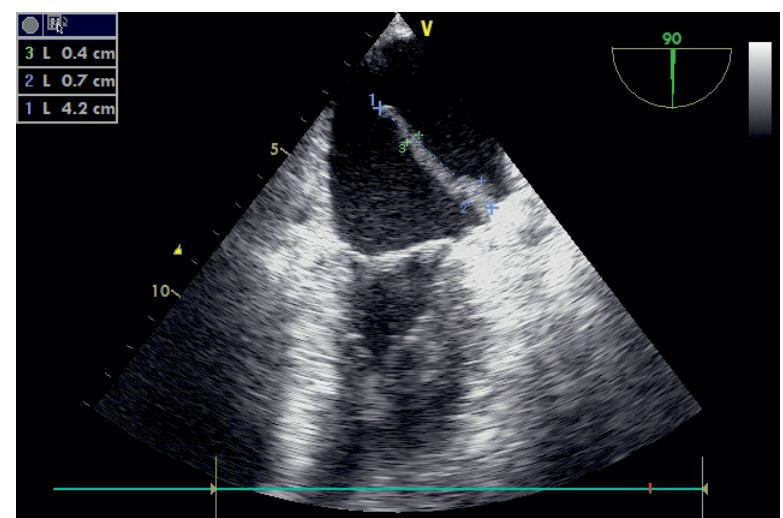

B

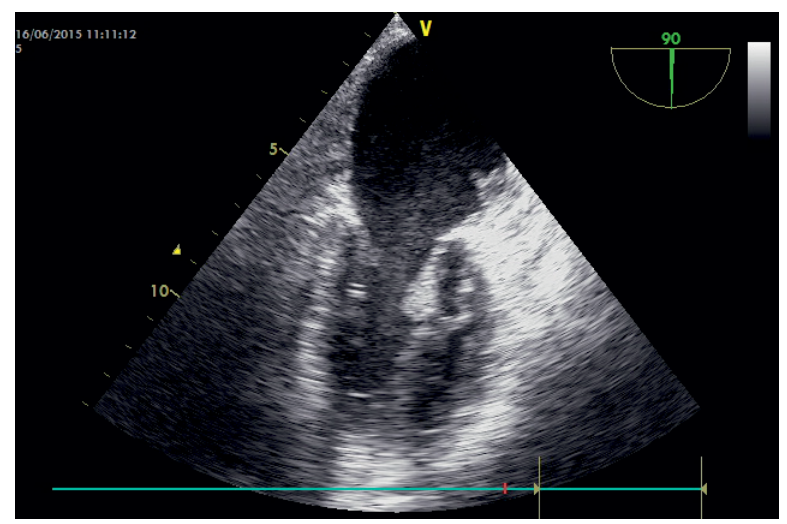

Figure 4. A huge $(4.2 \mathrm{~cm} \times 0.7 \mathrm{~cm})$, mobile thrombus on the atrial side of the Watchman ${ }^{\mathrm{TM}}$ device protruding across the mitral valve into the left ventricle (further examination at 6 months after implantation). Transesophageal echocardiography: mid-esophageal views at 90 degrees (4A and 4B)

antiplatelet and antithrombotic regimen comprises: aspirin $100 \mathrm{mg}$ q.s. started before the intervention, an intravenous infusion of heparin during the procedure, followed by short-term OA with warfarin (for 45 days) and long-term DAPT (for 6 months) [1].

Device-associated thrombus, mostly non-mobile, was observed in $4.2 \%$ of 478 patients successfully treated with WD implantation in the PROTECT AF trial [2] and in $1.1 \%$ of 90 patients who underwent WD implantation on DAPT in the Canadian multi-center registry [10]. Additionally, Kubo et al. identified LA thrombus (usually non-mobile, smaller than $2.0 \mathrm{~cm}$ ) attached to the WD in $3.4 \%$ of 119 implanted patients [9]. In this study, follow-up TEE examinations were performed 45 days, 6 months or 12 months after the procedure and device-related thrombosis usually occurred after OA discontinuation. Similarly to our patient, the prevalence of permanent AF in the study by Kubo et al. was more common and the mean $\mathrm{CHADS}_{2}$ score tended to be higher $(3.8 \pm 0.6$ vs. $2.5 \pm 0.1 ; p=0.06)$ in patients with device-associated thrombus than in those without it [9].

Consistently with a case report by Elabbassi et al. describing a 75-year-old woman with device-related thrombus overlying a LA occlusion device implanted in the presence of persistent spontaneous contrast despite prolonged OA, we observed spontaneous contrast in the LA in pre-procedural and follow-up TEE examinations in our patient [13]. In line with the findings of the above studies, it is recommended to perform TEE at 45 days to 6 months after the implantation, since most thrombi were documented so far at 45-day TEE [5].

In the majority of studies, warfarin therapy was restarted or continued for another 4-8 weeks after detection of device-related thrombus $[5,9,10]$. In some cases, low-molecular-heparin treatment may be also considered for another 4-8 weeks [5]. A control TEE examination is suggested to guide the decision regarding further management. This approach appears to be successful in the majority of patients, especially in those with small thrombus. As reported by Kubo et al. and Saw et al., the thrombus disappeared in all patients at follow-up TEE examination, mostly within 6 months after the identification of device-associated thrombus $[9,10]$. Therefore, anticoagulant therapy is recommended in all patients with device-associated thrombus regardless of the symptoms until thrombus resolution is confirmed by follow-up TEE [5]. As reported by Elabbassi et al. and observed in our patient, this kind of treatment regimen is not always successful, especially in patients with permanent AF, large LA and dense spontaneous echo contrast in TEE [13].

There is only little evidence on the association between the presence of device-attached thrombus and an increased incidence of thromboembolic events $[2,9,10]$. Acute stroke caused by air or thrombus embolization after implantation of the WD occurred in $1.1 \%$ of 478 procedures in the PROTECT AF trial during a mean follow-up of just more than a year [2]. In the study by Kubo et al. there were no death, stroke and systemic embolism events during a follow-up of $1456 \pm 546$ days [9]. In the Canadian multi-center registry, there was only 1 case of transient ischemic attack and no stroke or death during a mean follow-up of $173.8 \pm 117.3$ days [10]. Unexpectedly, the patient discussed in this case report remained completely asymptomatic despite the presence of large, mobile thrombus in the LA.

There is no strict recommendation in the EHRA/ /EAPCI expert consensus statement on the indications for cardiac surgery in patients with device-related thrombosis [5]. In our opinion, cardiac surgery should be considered in patients with device-associated late thrombosis, especially if the thrombus is mobile, greater than $2.0 \mathrm{~cm}$, with high risk of embolization, or in patients with a history of thromboembolic event. 
As in the described case, such decision must be made after taking into account individual risk factors present in the particular patient.

Despite the already obtained evidence on the effectiveness of percutaneous LAA closure, further studies are needed to evaluate the risk of late WD-related thrombosis and to investigate whether the common regimen with short-term OA, followed by long-term DAPT started already before the complete endothelization of the device is sufficient to prevent device-associated late thrombosis, especially in patients with high thromboembolic risk. Our case report exemplifies that device-associated late thrombosis may occur even during optimal long-term OA. Based on the observations by Kaul et al., we believe that non-vitamin $\mathrm{K}$ antagonist oral anticoagulants (NOACs) may constitute a valuable alternative in subjects undergoing WD implantation [14].

\section{Conclusions}

Device-associated thrombus formation in long-term follow-up after the implantation of the WD remains a rare but severe complication. Formal recommendations regarding prevention and management of device-related thrombosis are still lacking. Careful long-term echocardiographic monitoring seems reasonable after percutaneous LAA closure, especially in high thromboembolic risk patients (e.g. those with permanent $A F$, large LA and dense spontaneous echo contrast in echocardiography). Prolongation of OA with warfarin or treatment with NOACs after the implantation of WD should be considered individually in these high-risk patients. Finally, due to the paucity of evidence-based data, we suggest an individualized treatment approach in patients with device-associated thrombus after percutaneous LAA closure.

\section{References}

1. Holmes DR, Reddy VY, Turi ZG et al. Percutaneous closure of the left atrial appendage versus warfarin therapy for prevention of stroke in patients with atrial fibrillation: a randomized non-inferiority trial. Lancet 2009; 374: 534-542.

2. Reddy VY, Holmes DR, Doshi SK et al. Safety of percutaneous left atrial appendage closure: Results from the Watchman Left Atrial Appendage System for Embolic Protection in Patients With AF (PROTECT AF) Clinical Trial and the Continued Access registry. Circulation 2011; 123: 417-424.

3. Reddy VY, Mobius-Winkler S, Miller MA et al. Left atrial appendage closure with the Watchman device in patients with a contraindication for oral anticoagulation. J Am Coll Cardiol 2013; 61: 2551-2556.

4. Camm AJ, Lip GY, De Caterina R et al. 2012 focused update of the ESC Guidelines for the management of atrial fibrillation. Eur Heart J 2012; 33: 2719-2747.

5. Meier B, Blaauw $Y$, Khattab AA et al. EHRA/EAPCl expert consensus statement on catheter-based left atrial appendage occlusion. Eurointervention 2015; 10: 1109-1125.

6. Bajaj NS, Gaba S, Arora P et al. Meta-analysis of randomized control trials (RCT) comparing percutaneous left atrial appendage (Watchman device) closure versus adjusted dose warfarin for stroke prophylaxis in non-valvular atrial fibrillation. J Am Coll Cardiol 2015; 65 (10 S).

7. Mobius-Winkler S, Majunke N, Sandri M et al. Percutaneous left atrial appendage closure: Technical aspects and prevention of periprocedural complications with the Watchman device. World J Cardiol 2015; 7: 65-75.

8. Viles-Gonzales JF, Kar S, Douglas P et al. The clinical impact of incomplete left atrial appendage closure with the Watchman device in patients with atrial fibrillation. J Am Coll Cardiol 2012; 59: 923-929.

9. Kubo S, Mizutani Y, Tat E et al. Incidence, characteristics and clinical course of left atrial thrombus attached to Watchman device in atrial fibrillation patients. J Am Coll Cardiol 2015; 66 (15_S).

10. Saw J, Fahmy P, Azzalini L et al. Canadian multi-center experience with Watchman for percutaneous left atrial appendage closure. J Am Coll Cardiol 2015; 66 (15_S)

11. Lam SC, Bertog S, Sievert H. Incomplete left atrial appendage occlusion and thrombus formation after Watchman implantation treated with anticoagulation followed by further transcatheter closure with a second-generation Amplatzer Cardiac Plug (Amulet device). Catheter Cardiovasc Interv 2015; 85: 321-327.

12. Gasparini $M$, Ceriotti $C$, Bragato R. Huge left atrial thrombus after left atrial appendage occlusion with a Watchman device. Eur Heart $J$ 2012; 33: 1998.

13. Elabbassi W, Osman AN, Chowdhury MA et al. Device-related thrombus overlying a left-atrial occlusion device implanted for persistent apontaneous echo contrast despite prolonged oral anticoagulation therapy. Cardiology 2013; 125:78-81.

14. Kaul $S$, Cingolani $E$, Shehata $M$ et al. How does left atrial appendage occlusion using the Watchman device compare to dabigatran for reducing thromboembolic events in patients with nonvalvular atrial fibrillation? J Am Coll Cardiol 2010; 55 (suppl.10A): A4.E39. 